\title{
Isolation, Structure and Absolute Stereochemistry of Platensimycin, A Broad Spectrum Antibiotic Discovered Using an Antisense Differential Sensitivity Strategy
}

Sheo B. Singh, †* Hiranthi Jayasuriya, † John G. Ondeyka, † Kithsiri B. Herath, † Chaowei Zhang, $†$ Deborah L. Zink, $\uparrow$ Nancy N. Tsou, $\uparrow$ Richard G. Ball, $\uparrow$ Angela Basilio, $\ddagger$ Olga Genilloud, $\ddagger$ Maria Teresa Diez, $\ddagger$ Francisca Vicente, $\ddagger$

Fernando Pelaez, $\ddagger$ Katherine Young, $†$ Jun Wang†

Merck Research Laboratories, P. O. Box 2000, Rahway, New Jersey 07065 and CIBE, Merck Sharp \& Dohme de Espana, S. A. Josefa Valcárcel, Madrid, Spain

sheo_singh@merck.com

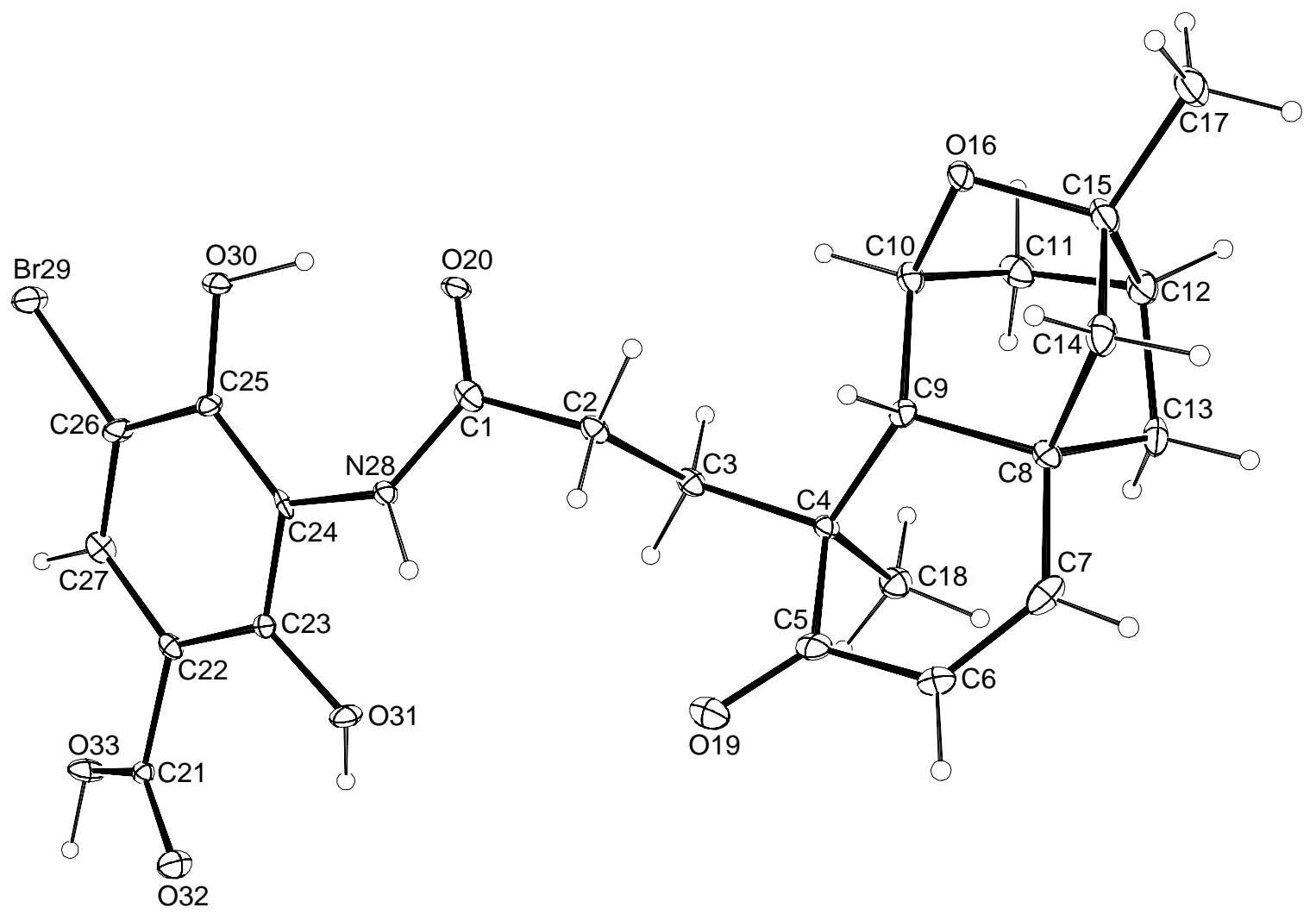

Figure S1. Perspective view (ORTEP) of platensimycin (1b), showing crystallographic numbering scheme. Nonhydrogen atoms are represented by ellipsoids corresponding to 50\% probability envelopes. Hydrogen atoms have been drawn at an arbitrary size. 


\section{Supporting Information}

Table S1. Crystal data and structure refinement for $\mathbf{1 b}$

\begin{tabular}{|c|c|}
\hline Formula & $\mathrm{C}_{24} \mathrm{H}_{26} \mathrm{Br} \mathrm{N} \mathrm{O} 7$ \\
\hline Fw & 520.360 \\
\hline Crystal colour & colorless \\
\hline Crystal dimen. (mm) & $0.06 \times 0.12 \times 0.20$ \\
\hline Lattice symmetry & orthorhombic \\
\hline Space group & $P 2{ }_{1}{ }_{1} 21$ \\
\hline$a,(\AA)$ & $10.176(4)$ \\
\hline$b$ & $11.376(5)$ \\
\hline C & $19.084(8)$ \\
\hline$\alpha$ & 90.00 \\
\hline$\beta$ & 90.00 \\
\hline$\gamma$ & 90.00 \\
\hline$V\left(\AA^{3}\right)$ & 2209.2(15) \\
\hline$Z$ & 4 \\
\hline$D_{\text {calc }}\left(\mathrm{Mg} \mathrm{m}^{-3}\right)$ & 1.564 \\
\hline Radiation $(K \alpha)$ & Mo \\
\hline Wavelength (Á) & 0.71073 \\
\hline Temperature (K) & 100 \\
\hline$\mu\left(\mathrm{mm}^{-1}\right)$ & 1.908 \\
\hline Diffractometer & Bruker PLATFORM geometry, Bruker SMART1K CCD \\
\hline Reflections measured & 24342 \\
\hline Resolution $(\AA)$ & 0.80 \\
\hline Unique reflections & 4561 \\
\hline Rint & 0.116 \\
\hline Flack Parameter & $-0.011(8)$ \\
\hline Reflections used & 4561 \\
\hline Refl. obsd. criterion & $>2 \sigma(I)$ \\
\hline Variables & 295 \\
\hline Refined on & $F^{2}$ \\
\hline$R$ (based on obsd. data) & 0.038 \\
\hline$R_{W}($ based on all data $)$ & 0.075 \\
\hline$S$ & 0.89 \\
\hline residual peak (e̊̊-3) & $0.50(8)$ \\
\hline \multicolumn{2}{|l|}{ Computer programs: } \\
\hline Solution & SHELXS-97 \\
\hline Refinement & SHELXL-97 \\
\hline
\end{tabular}


Supporting Information

Table S2. Bond Distances in Angstrom

$\begin{array}{ll}\text { Br29-C26 } & 1.878(4) \\ \mathrm{O} 16-\mathrm{C} 10 & 1.456(4) \\ \mathrm{O} 16-\mathrm{C} 15 & 1.466(4) \\ \mathrm{O} 19-\mathrm{C} 5 & 1.225(5) \\ \mathrm{O} 20-\mathrm{C} 1 & 1.248(4) \\ \mathrm{O} 30-\mathrm{C} 25 & 1.353(4) \\ \mathrm{O} 31-\mathrm{C} 23 & 1.366(4) \\ \mathrm{O} 32-\mathrm{C} 21 & 1.216(4) \\ \mathrm{O} 33-\mathrm{C} 21 & 1.294(4) \\ \mathrm{N} 28-\mathrm{C} 1 & 1.341(5) \\ \mathrm{N} 28-\mathrm{C} 24 & 1.441(4) \\ \mathrm{C} 1-\mathrm{C} 2 & 1.506(5) \\ \mathrm{C} 2-\mathrm{C} 3 & 1.528(5) \\ \mathrm{C} 3-\mathrm{C} 4 & 1.544(5) \\ \mathrm{C} 4-\mathrm{C} 5 & 1.542(5) \\ \mathrm{C} 4-\mathrm{C} 9 & 1.564(4) \\ \mathrm{C} 4-\mathrm{C} 18 & 1.555(5) \\ \mathrm{C} 5-\mathrm{C} 6 & 1.473(5) \\ \mathrm{C} 6-\mathrm{C} 7 & 1.336(6)\end{array}$

$\begin{array}{ll}\text { C7-C8 } & 1.496(5) \\ \text { C8-C9 } & 1.559(5) \\ \text { C8-C13 } & 1.569(5) \\ \text { C8-C14 } & 1.557(5) \\ \text { C9-C10 } & 1.551(5) \\ \text { C10-C11 } & 1.530(5) \\ \text { C11-C12 } & 1.539(5) \\ \text { C12-C15 } & 1.563(5) \\ \text { C12-C13 } & 1.546(5) \\ \text { C14-C15 } & 1.542(5) \\ \text { C15-C17 } & 1.527(4) \\ \text { C21-C22 } & 1.511(4) \\ \text { C22-C23 } & 1.397(5) \\ \text { C22-C27 } & 1.405(5) \\ \text { C23-C24 } & 1.415(5) \\ \text { C24-C25 } & 1.390(5) \\ \text { C25-C26 } & 1.427(5) \\ \text { C26-C27 } & 1.382(5)\end{array}$

Table S3. Bond Angles in Degrees

\begin{tabular}{|c|c|c|c|}
\hline $\mathrm{C} 10-\mathrm{O} 16-\mathrm{C} 15$ & $103.6(2)$ & $\mathrm{C} 13-\mathrm{C} 12-\mathrm{C} 15$ & 104.8(3) \\
\hline C1-N28-C24 & $130.4(3)$ & $\mathrm{C} 11-\mathrm{C} 12-\mathrm{C} 13$ & 107.9(3) \\
\hline N28-C1-C2 & 115.7(3) & $\mathrm{C} 11-\mathrm{C} 12-\mathrm{C} 15$ & 103.3(3) \\
\hline $\mathrm{O} 20-\mathrm{C} 1-\mathrm{N} 28$ & $123.0(3)$ & C8-C13-C12 & 100.7(3) \\
\hline $\mathrm{O} 20-\mathrm{C} 1-\mathrm{C} 2$ & 121.0(3) & $\mathrm{C} 8-\mathrm{C} 14-\mathrm{C} 15$ & 100.6(3) \\
\hline $\mathrm{C} 1-\mathrm{C} 2-\mathrm{C} 3$ & 109.1(3) & $\mathrm{O} 16-\mathrm{C} 15-\mathrm{C} 14$ & 105.8(3) \\
\hline $\mathrm{C} 2-\mathrm{C} 3-\mathrm{C} 4$ & 115.7(3) & $\mathrm{O} 16-\mathrm{C} 15-\mathrm{C} 17$ & 108.4(3) \\
\hline $\mathrm{C} 3-\mathrm{C} 4-\mathrm{C} 5$ & $110.5(3)$ & $\mathrm{O} 16-\mathrm{C} 15-\mathrm{C} 12$ & 104.7(3) \\
\hline C3-C4-C18 & 106.9(3) & $\mathrm{C} 12-\mathrm{C} 15-\mathrm{C} 17$ & 116.4(3) \\
\hline $\mathrm{C} 5-\mathrm{C} 4-\mathrm{C} 9$ & 106.5(3) & $\mathrm{C} 14-\mathrm{C} 15-\mathrm{C} 17$ & 115.8(3) \\
\hline C3-C4-C9 & 111.7(3) & $\mathrm{C} 12-\mathrm{C} 15-\mathrm{C} 14$ & 104.8(3) \\
\hline C9-C4-C18 & 116.1(3) & $\mathrm{O} 33-\mathrm{C} 21-\mathrm{C} 22$ & 113.1(3) \\
\hline $\mathrm{C} 5-\mathrm{C} 4-\mathrm{C} 18$ & $105.0(3)$ & O32-C21-O33 & $126.0(3)$ \\
\hline $\mathrm{O} 19-\mathrm{C} 5-\mathrm{C} 6$ & $122.5(3)$ & $\mathrm{O} 32-\mathrm{C} 21-\mathrm{C} 22$ & 120.9(3) \\
\hline $\mathrm{O} 19-\mathrm{C} 5-\mathrm{C} 4$ & $120.8(3)$ & C21-C22-C23 & 120.9(3) \\
\hline $\mathrm{C} 4-\mathrm{C} 5-\mathrm{C} 6$ & 116.7(3) & $\mathrm{C} 21-\mathrm{C} 22-\mathrm{C} 27$ & 120.5(3) \\
\hline $\mathrm{C} 5-\mathrm{C} 6-\mathrm{C} 7$ & 123.2(3) & $\mathrm{C} 23-\mathrm{C} 22-\mathrm{C} 27$ & 118.5(3) \\
\hline C6-C7-C8 & 124.0(3) & $\mathrm{O} 31-\mathrm{C} 23-\mathrm{C} 22$ & 124.8(3) \\
\hline $\mathrm{C} 7-\mathrm{C} 8-\mathrm{C} 14$ & 113.6(3) & $\mathrm{C} 22-\mathrm{C} 23-\mathrm{C} 24$ & 120.4(3) \\
\hline $\mathrm{C} 9-\mathrm{C} 8-\mathrm{C} 13$ & $112.0(3)$ & O31-C23-C24 & 114.8(3) \\
\hline $\mathrm{C} 9-\mathrm{C} 8-\mathrm{C} 14$ & 107.1(3) & N28-C24-C25 & $124.5(3)$ \\
\hline $\mathrm{C} 7-\mathrm{C} 8-\mathrm{C} 9$ & 111.0(3) & C23-C24-C25 & 120.7(3) \\
\hline $\mathrm{C} 7-\mathrm{C} 8-\mathrm{C} 13$ & 114.8(3) & N28-C24-C23 & 114.8(3) \\
\hline C13-C8-C14 & $97.5(2)$ & $\mathrm{O} 30-\mathrm{C} 25-\mathrm{C} 26$ & 116.6(3) \\
\hline C4-C9-C8 & 114.3(3) & $\mathrm{C} 24-\mathrm{C} 25-\mathrm{C} 26$ & 118.7(3) \\
\hline $\mathrm{C} 4-\mathrm{C} 9-\mathrm{C} 10$ & 118.1(3) & $\mathrm{O} 30-\mathrm{C} 25-\mathrm{C} 24$ & 124.8(3) \\
\hline C8-C9-C10 & 110.1(3) & $\mathrm{C} 25-\mathrm{C} 26-\mathrm{C} 27$ & $120.0(3)$ \\
\hline $\mathrm{O} 16-\mathrm{C} 10-\mathrm{C} 11$ & 100.3(3) & $\mathrm{Br} 29-\mathrm{C} 26-\mathrm{C} 25$ & 117.8(2) \\
\hline C9-C10-C11 & $114.4(3)$ & $\mathrm{Br} 29-\mathrm{C} 26-\mathrm{C} 27$ & 122.2(3) \\
\hline $\mathrm{O} 16-\mathrm{C} 10-\mathrm{C} 9$ & 106.9(3) & $\mathrm{C} 22-\mathrm{C} 27-\mathrm{C} 26$ & 121.6(3) \\
\hline C10-C11-C12 & 98.9(3) & & \\
\hline
\end{tabular}

Numbers in parentheses are estimated standard deviations in the least significant digits. 


\section{Supporting Information}

Table S4. Fractional Atomic Coordinates and Equivalent Isotropic Displacement Parameters

\begin{tabular}{|c|c|c|c|c|}
\hline & $x$ & $y$ & $Z$ & Ueq \\
\hline Br29 & $-0.36849(4)$ & 1.30828(3) & $-0.03899(2)$ & $0.02290(11)$ \\
\hline O16 & $0.3726(3)$ & $0.66462(19)$ & $-0.16560(12)$ & $0.0189(6)$ \\
\hline O19 & $-0.1579(2)$ & $0.5022(2)$ & $-0.09704(14)$ & $0.0238(7)$ \\
\hline O20 & $-0.0328(2)$ & $0.9557(2)$ & $-0.08961(13)$ & $0.0154(6)$ \\
\hline O30 & $-0.1754(2)$ & $1.11555(18)$ & $-0.03069(13)$ & $0.0173(6)$ \\
\hline O31 & $-0.4538(2)$ & $0.7879(2)$ & $-0.07888(12)$ & $0.0167(6)$ \\
\hline O32 & $-0.7169(2)$ & $0.8359(2)$ & $-0.06097(12)$ & $0.0209(7)$ \\
\hline O33 & $-0.7746(2)$ & $1.0172(2)$ & $-0.09452(14)$ & $0.0218(7)$ \\
\hline N28 & $-0.2166(3)$ & $0.8592(2)$ & $-0.05299(15)$ & $0.0117(7)$ \\
\hline C1 & $-0.0906(4)$ & $0.8627(3)$ & $-0.07337(19)$ & $0.0158(10)$ \\
\hline $\mathrm{C} 2$ & $-0.0251(3)$ & $0.7452(3)$ & $-0.08355(19)$ & $0.0134(9)$ \\
\hline C3 & $-0.0518(3)$ & $0.7017(3)$ & $-0.15793(17)$ & $0.0149(9)$ \\
\hline $\mathrm{C} 4$ & 0.0051(3) & $0.5799(3)$ & $-0.17633(19)$ & $0.0124(9)$ \\
\hline C5 & $-0.0552(4)$ & $0.4844(3)$ & $-0.1290(2)$ & $0.0171(10)$ \\
\hline C6 & $0.0149(4)$ & $0.3710(3)$ & $-0.1257(2)$ & $0.0183(10)$ \\
\hline $\mathrm{C} 7$ & $0.1372(4)$ & $0.3560(3)$ & $-0.14943(18)$ & $0.0213(9)$ \\
\hline C8 & $0.2201(3)$ & $0.4527(3)$ & $-0.1786(2)$ & $0.0155(9)$ \\
\hline C9 & $0.1572(3)$ & $0.5752(3)$ & $-0.16428(19)$ & $0.0130(9)$ \\
\hline C10 & $0.2426(3)$ & $0.6737(3)$ & $-0.19712(18)$ & $0.0163(9)$ \\
\hline C11 & $0.2770(4)$ & $0.6540(3)$ & $-0.27430(19)$ & $0.0211(10)$ \\
\hline C12 & $0.3572(4)$ & $0.5399(3)$ & $-0.26667(19)$ & $0.0208(9)$ \\
\hline C13 & $0.2595(4)$ & $0.4371(3)$ & $-0.2576(2)$ & $0.0203(10)$ \\
\hline C14 & $0.3618(4)$ & $0.4560(3)$ & $-0.1478(2)$ & $0.0217(9)$ \\
\hline C15 & $0.4239(3)$ & $0.5535(3)$ & $-0.1934(2)$ & $0.0198(10)$ \\
\hline C17 & $0.5735(3)$ & $0.5594(4)$ & $-0.1927(2)$ & $0.0314(12)$ \\
\hline C18 & $-0.0432(3)$ & $0.5495(3)$ & $-0.25143(19)$ & $0.0198(10)$ \\
\hline C21 & $-0.6912(3)$ & $0.9378(3)$ & $-0.07462(19)$ & $0.0106(9)$ \\
\hline C22 & $-0.5521(3)$ & $0.9834(3)$ & $-0.06866(18)$ & $0.0125(9)$ \\
\hline C23 & $-0.4454(4)$ & $0.9062(3)$ & $-0.06818(18)$ & $0.0123(9)$ \\
\hline C24 & $-0.3168(3)$ & $0.9485(3)$ & $-0.05580(18)$ & $0.0121(9)$ \\
\hline C25 & $-0.2941(3)$ & $1.0681(3)$ & $-0.0454(2)$ & $0.0135(8)$ \\
\hline C26 & $-0.4030(3)$ & $1.1468(3)$ & $-0.0488(2)$ & $0.0159(9)$ \\
\hline C27 & $-0.5283(4)$ & $1.1039(3)$ & $-0.05939(19)$ & $0.0167(10)$ \\
\hline Н30 & -0.0973 & 1.0621 & -0.0449 & 0.021 \\
\hline Н31 & -0.5307 & 0.7694 & -0.1004 & 0.020 \\
\hline H33 & -0.8591 & 0.9817 & -0.0938 & 0.026 \\
\hline H28 & -0.2415 & 0.7796 & -0.0395 & 0.014 \\
\hline H2A & -0.0600 & 0.6880 & -0.0492 & 0.016 \\
\hline H2B & 0.0707 & 0.7528 & -0.0760 & 0.016 \\
\hline Н3А & -0.0155 & 0.7599 & -0.1913 & 0.018 \\
\hline Н3В & -0.1481 & 0.6992 & -0.1651 & 0.018 \\
\hline H6 & -0.0291 & 0.3054 & -0.1056 & 0.022 \\
\hline H7 & 0.1737 & 0.2792 & -0.1477 & 0.026 \\
\hline H9 & 0.1677 & 0.5865 & -0.1126 & 0.016 \\
\hline H10 & 0.2031 & 0.7531 & -0.1893 & 0.020 \\
\hline H11A & 0.3304 & 0.7191 & -0.2936 & 0.025 \\
\hline H11B & 0.1976 & 0.6426 & -0.3035 & 0.025 \\
\hline H12 & 0.4215 & 0.5273 & -0.3056 & 0.025 \\
\hline H13A & 0.3022 & 0.3602 & -0.2661 & 0.024 \\
\hline H13B & 0.1827 & 0.4455 & -0.2890 & 0.024 \\
\hline H14A & 0.3615 & 0.4775 & -0.0975 & 0.026 \\
\hline H14B & 0.4075 & 0.3798 & -0.1540 & 0.026 \\
\hline H17A & 0.6030 & 0.6237 & -0.2231 & 0.047 \\
\hline H17B & 0.6096 & 0.4849 & -0.2099 & 0.047 \\
\hline H17C & 0.6042 & 0.5733 & -0.1448 & 0.047 \\
\hline
\end{tabular}

Singh et al ...Platensimycin......Supporting information

Page S4 of S7 
Supporting Information

\begin{tabular}{|c|c|c|c|c|}
\hline H18A & -0.0140 & 0.4701 & -0.2639 & 0.030 \\
\hline H18B & -0.0066 & 0.6061 & -0.2848 & 0.030 \\
\hline H18C & -0.1394 & 0.5530 & -0.2529 & 0.030 \\
\hline H27 & -0.6001 & 1.1572 & -0.0604 & 0.020 \\
\hline
\end{tabular}

$U e q=(1 / 3) \Sigma_{i} \Sigma_{j} U_{i j} a_{i}^{*} a_{j}^{*} \mathbf{a}_{i} \cdot \mathbf{a}_{j}$

Table S5. Anisotropic Displacement Parameters

\begin{tabular}{|c|c|c|c|c|c|c|}
\hline & $\begin{array}{l}\text { U11 } \\
\text { U12 }\end{array}$ & U22 & U33 & U23 & U13 & \\
\hline Br29 & $0.01554(18)$ & $0.01529(17)$ & $0.0379(2)$ & $-0.0018(2)$ & $-0.0025(2)$ & $-0.00109(19)$ \\
\hline O16 & $0.0091(13)$ & $0.0237(16)$ & $0.0240(15)$ & $-0.0107(12)$ & $-0.0024(14)$ & $0.0022(14)$ \\
\hline O19 & $0.0197(18)$ & $0.0272(16)$ & $0.0246(18)$ & $0.0024(14)$ & 0.0031(14) & $-0.0049(13)$ \\
\hline $\mathrm{O} 20$ & $0.0104(14)$ & $0.0133(15)$ & $0.0224(17)$ & $0.0013(13)$ & $0.0028(12)$ & $-0.0028(12)$ \\
\hline O30 & $0.0092(14)$ & $0.0162(13)$ & $0.0264(17)$ & $-0.0050(14)$ & $-0.0002(13)$ & $-0.0028(10)$ \\
\hline O31 & $0.0117(13)$ & $0.0148(15)$ & $0.0236(16)$ & $-0.0023(12)$ & $-0.0042(11)$ & $-0.0037(12)$ \\
\hline O32 & $0.0191(14)$ & $0.0165(15)$ & $0.0269(18)$ & $0.0011(12)$ & $-0.0019(12)$ & $-0.0018(12)$ \\
\hline O33 & $0.0104(14)$ & $0.0247(16)$ & $0.0304(18)$ & $0.0022(14)$ & $-0.0038(13)$ & $-0.0058(12)$ \\
\hline N28 & $0.0095(16)$ & $0.0111(15)$ & $0.014(2)$ & $0.0002(15)$ & $0.0007(15)$ & $0.0006(13)$ \\
\hline C1 & $0.014(2)$ & $0.024(2)$ & $0.010(2)$ & $0.0009(19)$ & $0.0000(17)$ & $0.0003(18)$ \\
\hline $\mathrm{C} 2$ & $0.012(2)$ & $0.014(2)$ & $0.014(2)$ & $0.0041(17)$ & $0.0022(18)$ & $0.0007(16)$ \\
\hline C3 & $0.0102(19)$ & $0.019(2)$ & $0.015(2)$ & $0.001(2)$ & $-0.0015(16)$ & 0.0011(19) \\
\hline $\mathrm{C} 4$ & $0.009(2)$ & $0.015(2)$ & $0.013(2)$ & $-0.0024(18)$ & 0.0000(18) & $-0.0009(16)$ \\
\hline $\mathrm{C} 5$ & $0.016(2)$ & $0.021(2)$ & $0.014(2)$ & $-0.003(2)$ & $-0.0038(19)$ & $-0.0062(19)$ \\
\hline C6 & $0.020(2)$ & $0.016(2)$ & $0.019(3)$ & $0.0020(19)$ & $-0.001(2)$ & $-0.0050(18)$ \\
\hline $\mathrm{C} 7$ & $0.030(2)$ & $0.0140(19)$ & $0.019(2)$ & $-0.0020(17)$ & $-0.009(2)$ & $0.001(2)$ \\
\hline C8 & $0.010(2)$ & $0.016(2)$ & $0.020(3)$ & $-0.0004(19)$ & $-0.0012(18)$ & $0.0011(17)$ \\
\hline C9 & $0.013(2)$ & $0.016(2)$ & $0.010(2)$ & $-0.0045(17)$ & $-0.0035(18)$ & $0.0000(17)$ \\
\hline C10 & $0.013(2)$ & $0.016(2)$ & $0.020(2)$ & $0.000(2)$ & $0.0003(18)$ & $0.0035(17)$ \\
\hline C11 & $0.018(2)$ & $0.030(3)$ & $0.015(2)$ & $0.0000(19)$ & $0.0015(19)$ & $-0.0024(19)$ \\
\hline C12 & $0.018(2)$ & $0.029(2)$ & $0.015(2)$ & $-0.0075(18)$ & $0.000(2)$ & $0.000(2)$ \\
\hline C13 & $0.018(2)$ & $0.023(2)$ & $0.020(3)$ & $-0.010(2)$ & $-0.003(2)$ & $0.0079(19)$ \\
\hline C14 & $0.019(2)$ & $0.022(2)$ & $0.024(2)$ & $-0.0030(18)$ & $-0.003(2)$ & $0.008(2)$ \\
\hline C15 & $0.012(2)$ & $0.026(2)$ & $0.021(3)$ & $-0.003(2)$ & 0.0017(19) & $0.0038(18)$ \\
\hline C17 & $0.014(2)$ & $0.043(3)$ & $0.038(3)$ & $-0.017(2)$ & $0.000(2)$ & $0.004(2)$ \\
\hline C18 & $0.018(2)$ & $0.020(2)$ & $0.021(3)$ & $0.000(2)$ & $-0.002(2)$ & $0.000(2)$ \\
\hline C22 & $0.012(2)$ & $0.017(2)$ & $0.009(2)$ & $0.0018(17)$ & $0.0016(17)$ & 0.0001(17) \\
\hline C23 & $0.016(2)$ & $0.016(2)$ & $0.005(2)$ & $-0.0021(17)$ & $0.0032(17)$ & $-0.0048(17)$ \\
\hline C24 & $0.0076(19)$ & $0.019(2)$ & $0.009(2)$ & $-0.0003(18)$ & $0.0032(16)$ & 0.0039(16) \\
\hline C25 & 0.0137(19) & $0.0158(19)$ & $0.011(2)$ & $-0.003(2)$ & $-0.0006(19)$ & $-0.0047(16)$ \\
\hline C26 & $0.018(2)$ & $0.0072(17)$ & $0.023(3)$ & $0.0031(19)$ & 0.0003(19) & $-0.0018(15)$ \\
\hline $\mathrm{C} 27$ & $0.015(2)$ & $0.019(2)$ & $0.016(3)$ & $0.0053(18)$ & $0.0001(18)$ & 0.0036(17) \\
\hline
\end{tabular}


$125 \mathrm{MHz}{ }^{13} \mathrm{C}$ NMR spectrum of platensimycin in $\mathrm{C}_{5} \mathrm{D}_{5} \mathrm{~N}$

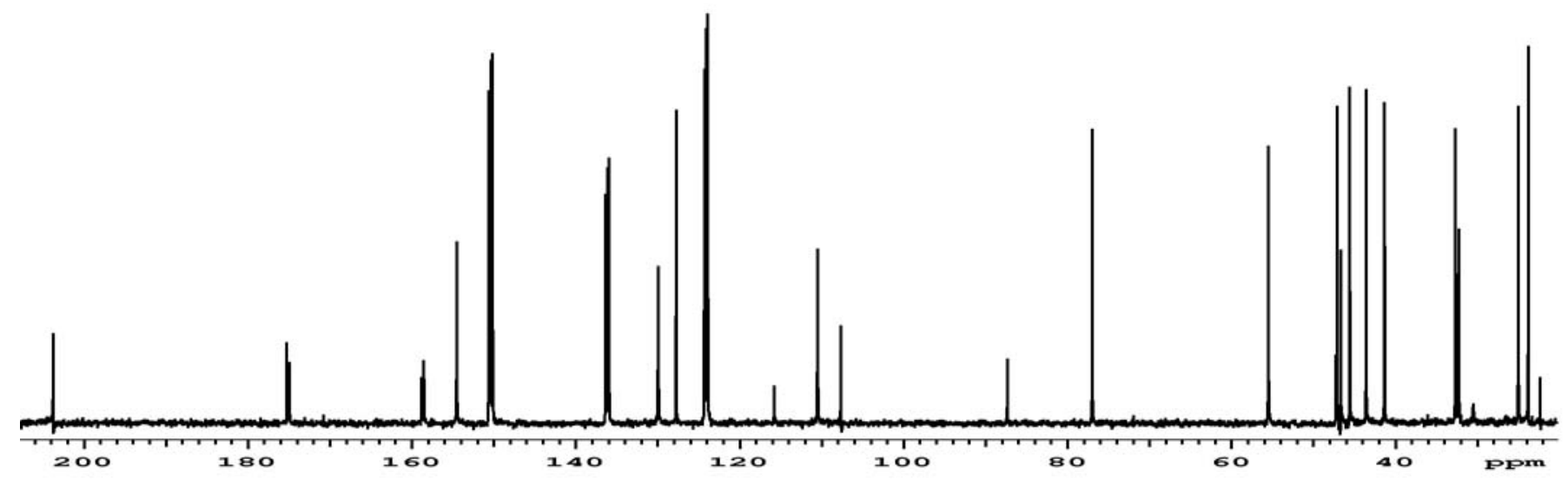

$500 \mathrm{MHz}{ }^{1} \mathrm{H}$ NMR spectrum of platensimycin in $\mathrm{C}_{5} \mathrm{D}_{5} \mathrm{~N}$

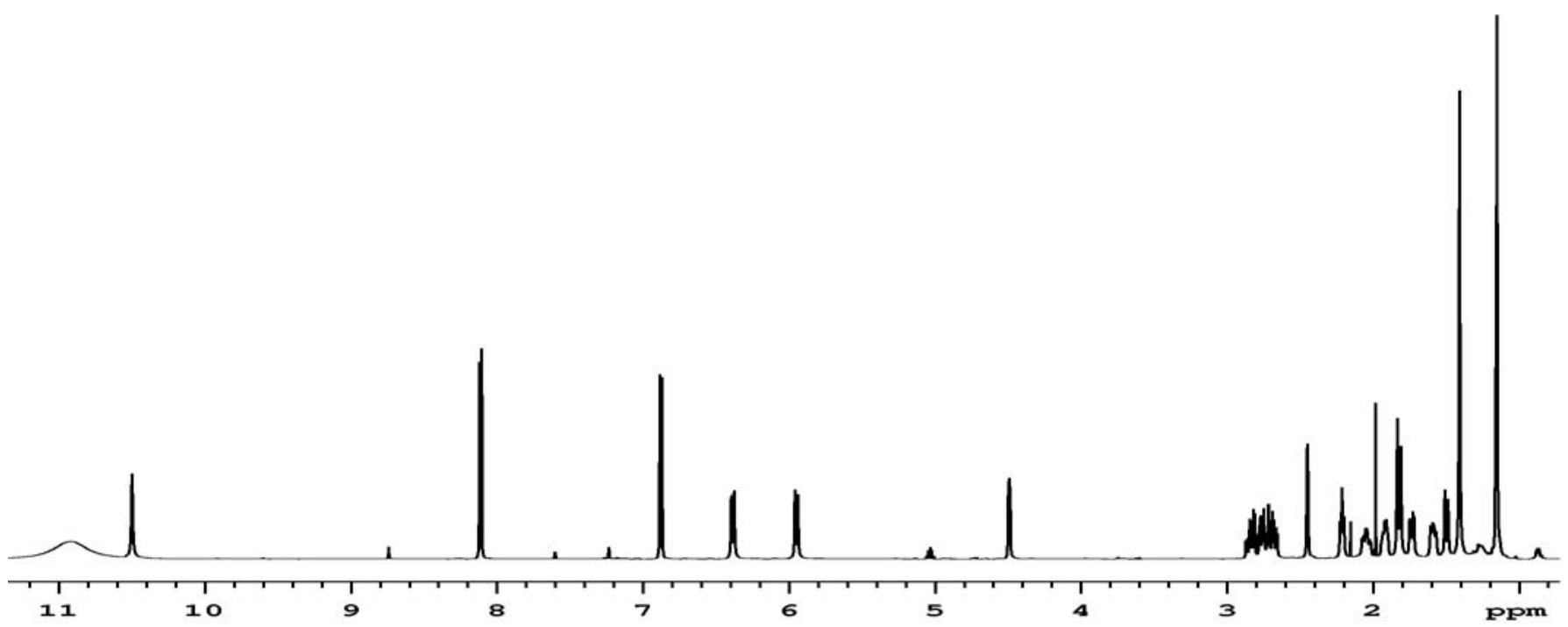




\section{Platensimycin (HPLC/UV) \\ Zorbax RX C 8 (4.6 x $250 \mathrm{~mm})$ \\ 20 to $95 \%$ aq $\mathrm{CH}_{3} \mathrm{CN}+0.1 \%$ TFA in $15 \mathrm{~min}, 1 \mathrm{~mL} / \mathrm{min}$}
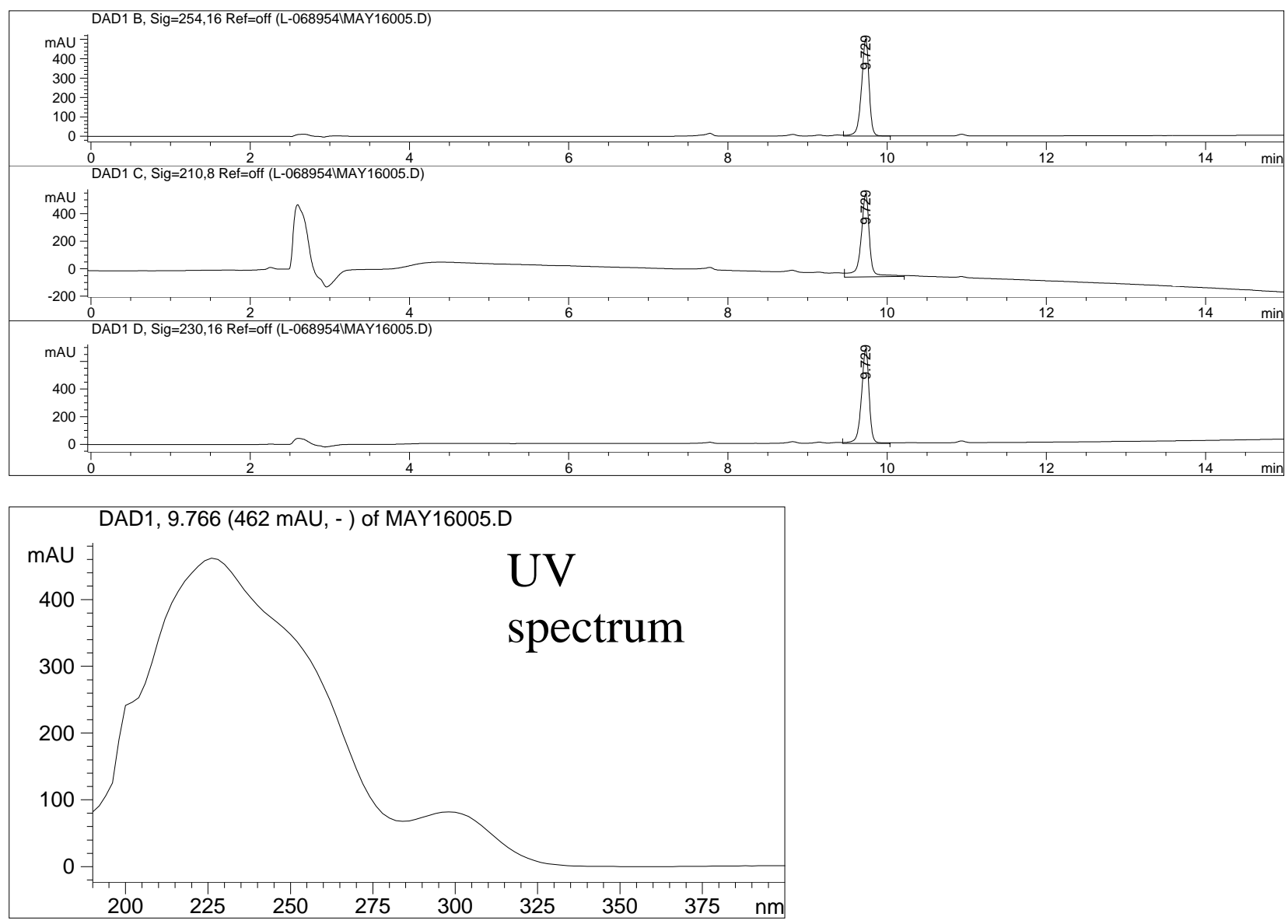

References with complete list of authors:

(6) Kodali, S.; Galgoci, A.; Young, K.; Painter, R.; Silver, L. L.; Herath, K. B.; Singh, S. B.; Cully, D.; Barrett, J. F.; Schmatz, D.; Wang, J. J. Biol. Chem. 2005, 280, 1669-1677.

(7) Young, K.; Jayasuriya, H.; Ondeyka, J. G.; Herath, K.; Zhang, C.; Kodali, S.; Galgoci, A.; Painter, R.; Brown-Driver, V.; Yamamoto, R.; Silver, L. L.; Zheng, Y.; Ventura, J. I.; Sigmund, J.; Ha, S.; Basilio, A.; Vicente, F.; Tormo, J. R.; Pelaez, F.; Youngman, P.; Cully, D.; Barrett, J. F.; Schmatz, D.; Singh, S. B.; Wang, J. Antimicrob. Agents Chemother. 2006, 50, 519-526.

(8) Wang, J.; Soisson, S. M.; Young, K.; Shoop, W.; Kodali, S.; Galgoci, A.; Painter, R.; Parthasarathy, G.; Tang, Y.; Cummings, R.; Ha, S.; Dorso, K.; Motyl, M.; Jayasuriya, H.; Ondeyka, J.; Herath, K.; Zhang, C.; Hernandez, L.; Alloco, J.; Basilio, Á.; Tormo, J. R.; Genilloud, O.; Vicente, F.; Pelaez, F.; Colwell, L.; Lee, S. H.; Michael, B.; Felcetto, T.; Gill, C.; Silver, L. L.; Hermes, J.; Bartizal, K.; Barrett, J.; Schmatz, D.; Becker, J. W.; Cully, D.; Singh, S. B. Nature 2006, 441, 358-361. 